\title{
Balance of Polarization in a Hybrid Fiber Optic Sensor
}

\author{
M. ŻYCZKOWski*, M. Szustakowski, P. Markowski and M. Karol \\ Military University of Technology, Institute of Optoelectronics, S. Kaliskiego 2, 00-908 Warsaw, Poland \\ This paper presents the effect of light polarization in a hybrid fiber optic sensor. The hybrid sensor is defined \\ as a combination of interferometer sensor and modalmetric sensor. Hybrid sensor system is based on the effect of \\ outputting interferometric sensor of interference as a result of changes made by the modalmetric system. Balance of \\ polarization in the arms of classical interferometer sensor leads to improvement of contrast of interference pattern \\ at the output of the interferometer. Aim of this study is to present a balance light polarization effects in a hybrid \\ system.
}

DOI: $10.12693 /$ APhysPolA.124.610

PACS: 42.81.Pa, 42.30.Wb, 42.79.Qx

\section{Design and operation of examined system}

This paper describes the effect of light polarization in the function of the hybrid Mach-Zender interferometer. Hybrid interferometers systems are the subject of research of many groups of researchers from different disciplines. Hybrid system combines the features of interference fiber optic sensor and modalmetric fiber optic sensor [1]. Modalmetric fiber sensor in hybrid system replaces the entire or a part of one arm of the interferometer. At the output of the hybrid system, interference pattern is recorded in the same way as in the classical interferometer. The relationship between the optical path difference between the arms of the interferometer can be expressed according to the following formula (1):

$$
\Delta \Phi=\frac{2 \pi}{\lambda_{0}} \Delta L,
$$

where $\Delta \Phi-$ phase shift, $\Delta L-$ optical path length shift, $\lambda_{0}$ - wavelength.

In the fiber optic interferometry, interference fringes are observed as a change of the intensity of the radiation in time. This change is a result of two waves, and the intensity of the radiation at the output of the interferometer can be described by Eq. (2):

$$
I_{\mathrm{OUT}}=\left\langle E E^{*}\right\rangle,
$$

where

$$
E=E_{1}+E_{2},
$$

$E_{1}, E_{2}$ - amplitude vectors of light propagating in the first and second arm of the interferometer.

A measure specifying the interference is contrast of interference fringes. Interference contrast can be described by the formula (4):

$$
V=\frac{I_{\max }-I_{\min }}{I_{\max }+I_{\min }}
$$

where $I_{\max }$ - maximum light intensity, $I_{\min }-$ minimum light intensity.

\footnotetext{
*corresponding author; e-mail: mzyczkowski@wat.edu.pl
}

Maximum possible radiation intensity is obtained if two waves of two interferometer arms are in phase while the minimum possible intensity is achieved when the two waves are out of phase. In other cases, the radiation intensity is a function of both waves amplitude vectors. In the case of light waves critical dependence on obtaining any interference is the state of polarization of the two waves. Polarization is a measure of the oscillation of propagating wave. Polarization states of light can be visualized in many ways, one of them is to determine point of the sphere known as the Poincaré sphere [2]. Each point on the sphere represents a distinct state of polarization, which can be described by two angular values.

The Poincaré sphere can contain several characteristic points. The poles represent the linear polarization state, while the equator of sphere represents the circular polarization states. All the remaining points on the sphere are all possible intermediate states of elliptical polarization. In the case of fiber-optic interferometry, critical value of the polarization are orthogonal states for which it is impossible to obtain an interference image. Interference fringes with a maximum amplitude can be achieved for the waves with two similar polarization states. If the polarization of the two waves is not uniform and different from the orthogonal interference, obtained pattern has a reduced amplitude in proportion to the difference in polarization states of both waves.

The above description applies to the interference system. In the hybrid system output signal is also dependent on the response of the modalmetric system. Modalmetric part of sensor is a system integrating lateral distribution of radiation. The connection between the multimode fiber and single-mode fiber is performed by an integrator of the specle pattern of radiation. Specle pattern of the radiation propagating in the fiber may be described by the formula (5):

$$
I(x, y, z)=\lim _{T \rightarrow \infty} \frac{1}{T} \int_{-T / 2}^{T / 2}|u(x, y, z, t)|^{2} \mathrm{~d} t=|A(x, y, z)|^{2},
$$

where $x, y, z$ - geometric dimensions of the fiber.

When the external disturbance occurs, the transverse distribution of radiation changes and the transfer of prop- 
agated optical power between excited modes or excitation of higher order modes proceeds. Observation of the segment of transverse distribution at the time causes that when the disorder occurs, the intensity of radiation on the part of the cross-section also changes (quantitatively). This type of event is observed at the output, as a direct change in the amplitude of radiation depends on the occurrence of disturbances.

The combination of the interference system with the modalmetric system causes that the output signal carries information consistent with the operation of the interferometric and modalmetric sensor. The operation of the modalmetric sensor significantly affects the interference pattern by introducing the direct amplitude changes corresponding with the nature of external disturbances. Direct change of the amplitude of the signal propagating in one interferometer arm caused by a disorder, deteriorates the visibility of interference pattern giving direct information about a disturbance. In this way, in a hybrid system, it is possible to precisely measure changes in the signal generated by the modalmetric system. This leads to increased sensitivity and speed of operation of the entire system.

\section{Examined sensor system}

The system was built using standard optical components used in telecommunication. For construction purposes, the following components were used: single mode of $9 \mu \mathrm{m}$ core and $125 \mu \mathrm{m}$ cladding and multimode of $62.5 \mu \mathrm{m}$ core and $125 \mu \mathrm{m}$ cladding. As a light source, a laser-C5-1550 Series of multi quantum well (MQW) distributed feedback (DFB) was applied, and as a detector standard telecommunications diode. The electrical signal was processed by the oscilloscope DLM 2054 Yokogawa. As disorder generator, a piezoelectric modulator with $8 \mathrm{~m}$ of multimode coiled fiber was used, and the modulator was triggered by the generator AFG3021 Tektronix. The Mach-Zehnder interferometer system consisted of two arms - reference arm with a length of $800 \mathrm{~m}$ made with single-mode fiber and a measuring arm with the length of $800 \mathrm{~m}$ made with three sections of single-mode fiber at $250 \mathrm{~m}$ and $50 \mathrm{~m}$ section of a multimode optical fiber. A diagram of the system is presented in Fig. 1.

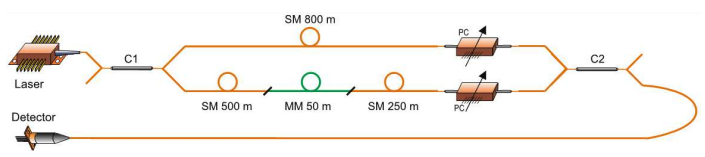

Fig. 1. The experimental setup.

Part of sensing structure for this type of sensor is a part of the measurement arm built with a multimode fiber.

The test system was equipped with a General Photonics electric polarization controller in order to compensate changes in polarization between the arms of the interferometer.

\section{Experimental details and results}

The system was tested for response to various types of signals applied to the piezo modulator. The test signals was:

- $1 \mathrm{~Hz}$ sine wave, presented in Fig. 2;

- $1 \mathrm{~Hz}$ rectangular wave, presented in Fig. 3;

- $1 \mathrm{~Hz}$ triangular wave, presented in Fig. 4;

- $1 \mathrm{~Hz}$ Lorentz wave, presented in Fig. 5.

The system was tested in terms of response for the matched polarization states between the two arms of the interferometer, and the lack of matched polarization. The results are presented in Figs. 2-5.

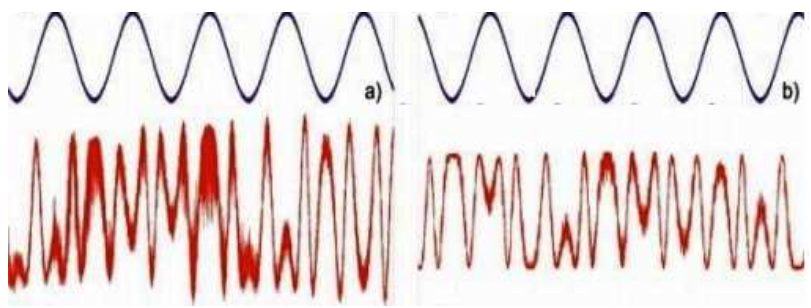

Fig. 2. The system response (red) to force a $1 \mathrm{~Hz}$ sine wave, (a) without matching polarization, (b) with matching polarization in both arms of the interferometer. Reference signal is marked with blue colour.

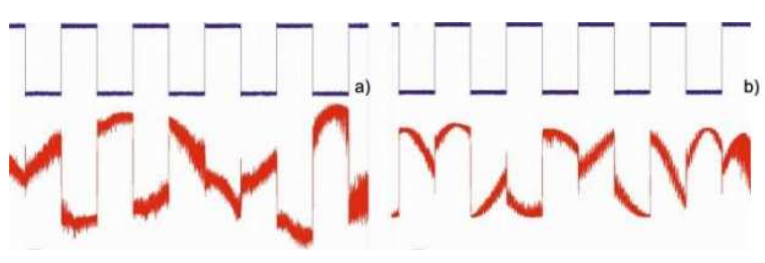

Fig. 3. As in Fig. 2, but for rectangular wave.

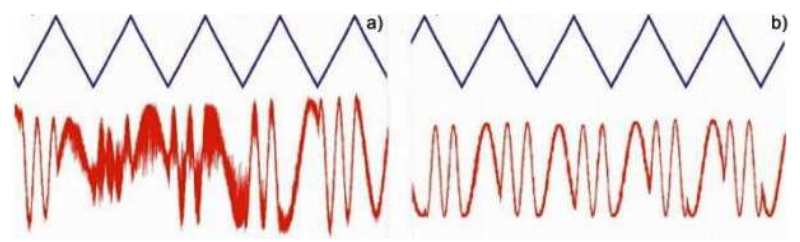

Fig. 4. As in Fig. 2, but for triangular wave.

The tested system was examined in terms of sensitivity to the frequency range $1 \mathrm{~Hz}-1 \mathrm{kHz}$. Characteristics of sensitivity as a function of frequency are presented in Fig. 6.

As presented in Fig. 6, polarization matching in both arms of interferometer is essential for high sensitivity of the hybrid system. 


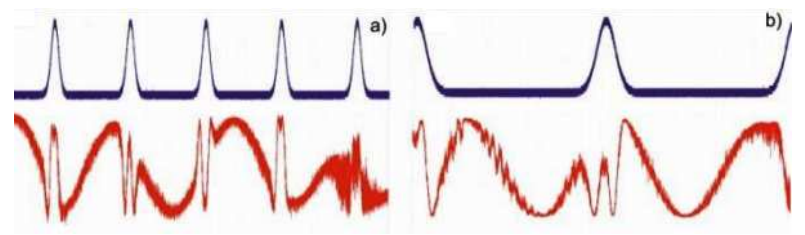

Fig. 5. As in Fig. 2, but for a Lorentz wave.

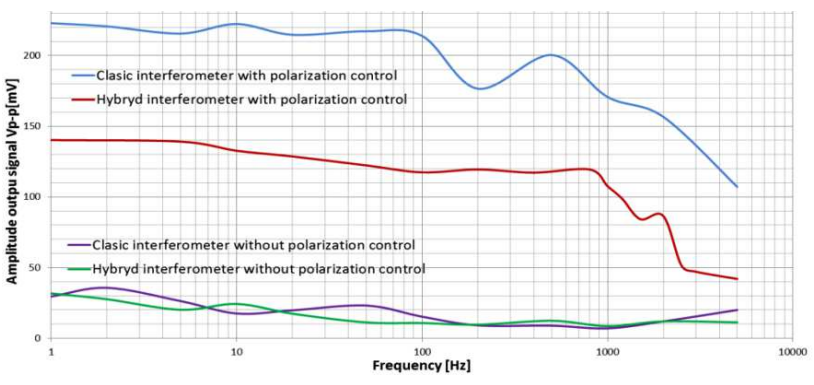

Fig. 6. Characteristics of sensitivity as function of frequency.

The final stage of the investigations was to measure the response time of the system. For this purpose, as a disorder a square wave signal was used. The measured parameter was the rise time defined as the time between the achievement of $10 \%$ and $90 \%$ of the maximum amplitude of the signal. The results are presented in graphic form in Fig. 7.

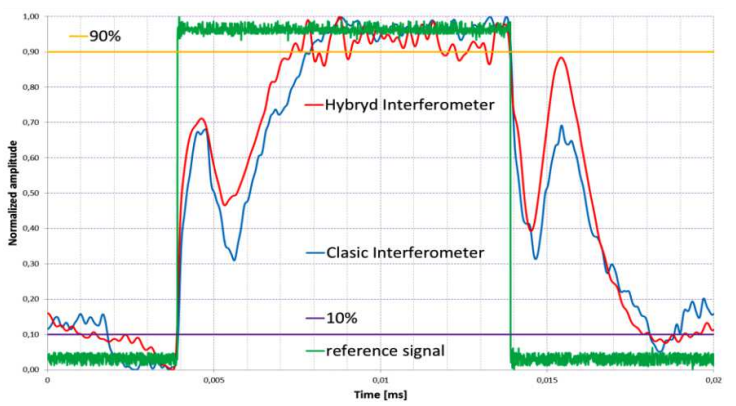

Fig. 7. Sensor response to rectangular stimulation.

Numerical results for the measurement of the response time are presented in Table.

\section{Conclusions}

Analyzing the results, especially the characteristics of the sensitivity of the hybrid system one can conclude that matching polarized waves propagating in the two arms of the interferometer is essential for the entire system. In the hybrid system an interferometric measure- ment leads to intensification of the response of modalmetric system. In the output signal, direct amplitude response of modalmetric sensor is modulated by interference image (fringes). This fact is a reason that during the TABLE

The timing parameters of hybrid and classical interferometer.

\begin{tabular}{c|c|c}
\hline \hline \multirow{2}{*}{ Amplitude } & \multicolumn{2}{|c}{ Time [ms] } \\
\cline { 2 - 3 } & Hybrid & Classic \\
\hline 10\% of $\max$ & 3.888 & 3.89 \\
90\% of $\max$ & 7.352 & 7.904 \\
rise time T90\%-T10\% & 3.464 & 4.014
\end{tabular}

occurrence of disturbances, an interference image is immediately changed and the change is proportional to the appearing disturbance. However, matching of the state of polarization waves of the two interferometer arms also increases the speed of system response. In this case, the interferometer generates better quality interference image to provide more accurate response of modalmetric system. Applying hybrid systems to known fiber optic sensors structures [3-11] with matching of polarization causes quicker reaction and identification of the external disturbance. This method of improving the output sensor signal can provide even more accurate localization of the disturbance and identification of the type of disorder. These improvements can be used to eliminate false alarms caused by environmental sources.

\section{References}

[1] M. Jedrzejewska-Szczerska, M. Gnyba, B.B. Kosmowski, Acta Phys. Pol. A 120, 621 (2011).

[2] S. Pancharatnam, Proc. Ind. Acad. Sci.-Section A 44, 247 (1956).

[3] K. Gut, K. Nowak, Europ. Phys. J. Spec. Top. 154, 89 (2008).

[4] M. Zyczkowski, M. Szustakowski, P. Markowski, M. Karol, Acta Phys. Pol. A 122, 938 (2012).

[5] K. Barczak, T. Pustelny, D. Dorosz, J. Dorosz, Acta Phys. Pol. A 114, A3 (2008).

[6] K. Gut, Acta Phys. Pol. A 114, A121 (2008).

[7] K. Barczak, Bull. Pol. Acad. Sci., Techn. Sci. 59, 409 (2011).

[8] M. Kondrat, M. Szustakowski, N. Pałka, W. Ciurapiński, M. Zyczkowski, Opto-Electron. Rev. 15, 127 (2007).

[9] M. Zyczkowski, M. Kondrat, W. Ciurapinski, J. Phys. IV: JP (France) 129, 189 (2005).

[10] M. Szustakowski, M. Chojnacki, M. Zyczkowski, N. Pałka, Opto-Electron. Rev. 9, 413 (2001).

[11] K. Gut, S. Drewniak, Acta Phys. Pol. A 120, 630 (2011). 